\title{
Discussed on a New Form of Resistivity Response Equation for Sandstone
}

\author{
Yang Kebing ${ }^{1, ~ *, ~ L i u ~ H u a n ~}{ }^{2}$, Cao Cheng Cheng ${ }^{1}$, Wu Xiaoning ${ }^{1}$ \\ ${ }^{1}$ Petrochina Huabei Oilfield Company, Renqiu, China \\ ${ }^{2}$ Zhangjiakou University, School of Tourism and Environment, Zhangjiakou, China
}

Email address:

ykbyzm123@sina.com (Yang Kebing)

${ }^{*}$ Corresponding author

\section{To cite this article:}

Yang Kebing, Liu Huan, Cao Cheng Cheng, Wu Xiaoning. Discussed on a New Form of Resistivity Response Equation for Sandstone. International Journal of Oil, Gas and Coal Engineering. Vol. 8, No. 6, 2020, pp. 130-136. doi: 10.11648/j.ogce.20200806.12

Received: October 19, 2020; Accepted: October 29, 2020; Published: November 9, 2020

\begin{abstract}
The resistivity response equation of sandstone is the most important basis for well logging interpretation to determine the water saturation of reservoir, but the Archie formula and its derivations are all empirical formulas, and the test error by experimental data is more than $20 \%$. Because the coincidence rate of the log interpretation of low resistivity oil layers and high resistivity water layers is low in the current, In order to further improve the calculation accuracy of reservoir water saturation, it is particularly necessary to deeply understand the essence of rock conductivity. Based on the main influencing factors of rock conductivity, It is considered that for rocks with given formation water resistivity and porosity, the influence of pore structure, size, argillaceous content and conductive minerals on rock conductivity is constant and is an inherent factor. rock resistivity only changes with the change of connected pore water volume, Therefore, the establishment of the relationship between rock resistivity and connected pore water volume conforms to the functional relationship form of dependent variable and independent variable, and can reflect the nature of the rock-electricity relationship. On this basis, the model of rock conductivity per unit volume is proposed, the response equation of sandstone resistivity is deduced, and the general form of the response equation of sandstone resistivity is obtained. The results show that the formula is in good agreement with the experimental data, with a correlation coefficient of more than 0.99 , and the test error of the experimental data is no more than $5 \%$, which can represent the theoretical form of the rock resistivity response equation. The new formula considers that rock resistivity and connected pore water volume or water saturation are power functions, which solves some obscure problems in rock resistivity response equation for many years. The parameters of the formula are the inherent property parameters of rocks, which have certain value in use. Based on the new formula, the formula of calculating the water saturation of a reservoir by using the ratio of radial resistivity has been obtained in practical application to some extent. it shows that the new formula is not only of theoretical significance, but also of practical application significance, and is worth further popularizing.
\end{abstract}

Keywords: Rock Resistivity, Connected Water Pore Volume, Response Equation, Derivation Proof, Attribute Parameter

\section{Introduction}

Resistivity is a physical quantity used to express the resistance characteristics of various substances. The resistance of a conductor of 1 meter in length and 1 square meter in cross-sectional area made of a certain material is numerically equal to the resistivity of this material. It reflects the blocking effect of a substance on the current. It depends on the type of substance and is affected by temperature. It can also be expressed as the resistance per unit volume of matter. In most cases, it is an intrinsic property parameter of a substance with a fixed value under certain temperature conditions. However, for all kinds of sandstones, the resistivity is not a fixed value, but varies with the pore water, argillaceous, conductive mineral and so on in the rock. Because the change of oil, gas and water in the connected pores of a reservoir can affect the change of rock resistivity, rock resistivity is one of the most critical parameters for evaluating the oil saturation of a reservoir.

Over the years, many methods [1-5] represented by Archie formula for calculating reservoir water saturation have been 
formed, and some models for evaluating reservoir resistivity have been formed, such as the three-water model [6, 7]. However, the research on the conductive mechanism of sandstone resistivity and the derivation of the calculation formula are quite complicated, the theoretical derivation and proof logic are not rigorous, and the practical application is often inconsistent. These methods cannot effectively evaluate low-resistivity reservoirs, complex pore reservoirs and water-flooded reservoirs [8-11]. The formulas formed to calculate water saturation are all empirical formulas, which are tested by experimental data and have a large error, such as Archie's formula, whose error sometimes exceeds $20 \%$. It shows that these formulas do not reflect the essence of rock conductivity, and are not the real rock conductivity formula [12-14].

Therefore, further research on the response equation of sandstone resistivity, revealing the conductive essence of rock, is helpful to improve the calculation accuracy of water saturation, and provide reliable parameters for reservoir evaluation and reserve calculation.

\section{Analysis of the Main Influencing Factors of Rock Resistivity}

Rocks are composed of minerals and pore fluids as well as cements, Most sandstones, when they do not contain conductive fluid, the rock skeletons made of rock-forming minerals are almost nonconductive. Many rocks conduct electricity because they have pores in the ground to varying degrees and fill them with a certain amount of saline solution. These salt solutions form positive ions (such as $\mathrm{Na}^{+}, \mathrm{Ca}^{2+}$, $\mathrm{Mg}^{2+}$, etc.) and negative ions (such as $\mathrm{C1}^{-}, \mathrm{SO}^{2-}$, etc.) due to the dissociation of salts, ions move under the action of electric fields, which form the vectors of current flow in rocks. As a result, an electric current flows through the pores water of the rock, and the rock becomes electrically conductive. Among them, the resistivity of metal minerals is very low, that of rock-forming minerals and petroleum is very high, that of igneous rocks is high, and that of sedimentary rocks is low. The variation of resistivity between different rocks and within the same rock is caused by many factors. It is generally believed that the main factors are porosity, pore structure, fluid properties contained in pores, argillaceous content, conductive minerals and temperature of rocks $[15,16]$.

The influence of porosity, pore structure and fluid properties in the pore on electrical conductivity is the most important factor. The greater the porosity of the rock, the simpler the pore structure, and the higher the salinity of the pore water contained, the lower the rock resistivity, and vice versa. There is also the question of whether the pore contains a full fluid. When the connected pores are full of water (water layer), the rock resistivity is relatively the lowest, while when the connected pores are full of oil (oil layer), the rock resistivity is relatively the highest. Therefore, for the same reservoir, the rock resistivity is a relative concept, which only indicates that the resistivity of the reservoir with oil content is higher than that of the reservoir with water content. Comparing with the resistivity of other reservoirs, it does not mean that the reservoir with high resistivity must be oil layer. This is also the fundamental reason why Archie's formula et al cannot evaluate low-resistivity oil layer and high-resistivity water layer when using the same rock-electric parameters for well logging interpretation [12-14].

The conductive mineral has the most obvious influence on the rock resistivity. When the rock contains conductive mineral bands (such as pyrite, etc.), the rock resistivity is extremely low. If there are non-uniformly distributed conductive mineral bands in the rock, the accurate evaluation of the rock resistivity will be affected. The argillaceous material in the rock is also conductive, but it does not rely on ions moving freely in the solution to transmit current. Instead, the ions absorbed by the argillaceous particles under the action of an external electric field move along the surface (usually cations) to transmit current. In addition, clay also adsorbed clay water and formed a conductive network to participate in the conductive process. The influence of argillaceous content on resistivity is reflected in the distribution of argillaceous bands or layered argillaceous materials and clays, which will lead to the reduction of rock resistivity $[17,18]$.

The existence of all the above influences requires connected conductive paths. When conductive minerals, argillaceous content and pore water are surrounded by the rock skeleton and no conductive network can be formed, it can be considered that these components do not participate in the conductive process without considering their influence on the conductivity of the rock.

It can be seen that the determining factor of rock conductivity depends on the conductive network formed during rock diagenesis, which includes the influence of porosity and pore structure. Except that changes in the volume of connected pore water will lead to changes in rock resistivity, for a given rock with a given formation water resistivity, all other factors are inherent influencing factors, that is, other factors are stable and cannot be changed, and only contribute fixed values (constants) to rock resistivity. Therefore, the change of rock resistivity is based on a base (inherent influencing factor), and the main influencing factor of rock resistivity change is the volume change of connected pore water. Based on this point of view, the establishment of the response relationship between rock resistivity and connected pore water volume (water saturation) will be able to effectively describe the characteristics of rock resistivity changes, and more clearly reflect the essence of rock conductivity. At the same time, in the response relationship, resistivity is the dependent variable, connected pore water volume or water saturation is the independent variable, and the functional relationship is clear. Compared with other empirical equations of rock resistivity, such as Archie formula, there are no problems of complex relationship, unclear concept and insufficient accuracy [12-14]. Moreover, the new formula is not an empirical formula, but can be derived from mathematical theory, which shows that the new formula is the best form of rock resistivity response equation. 


\section{Derivation of the Relationship Between Rock Resistivity and Connected Pore Water}

\subsection{The Establishment of Unit Volume Conductivity Model}

As mentioned in the previous analysis, the conductive system of sandstone is a connected network, which is actually composed of the pore space and the pore throat of the connected pore space. Because of the homogeneity of sedimentary sandstones, the concept of unit volume can be used to simulate and calculate the resistivity of sandstone. Assuming that a sandstone has a minimum conductive volume unit, which is called unit volume sandstone, the resistance of sandstone is the sum of the resistances in series and parallel of this unit volume sandstone. If the resistance of unit volume is $\mathrm{R}$, the resistance of sandstone can be expressed as:

$$
\frac{1}{R}=\sum_{i=1}^{i=n} \frac{1}{\sum_{k=1}^{k=n} r_{i k}}
$$

It can be seen that the sandstone unit volume model can simplify the derivation of resistivity formula. According to unit volume model, the conductive network can be designed as a parallel conductive mode of large throat connected pore water (free water), micro throat connected pore water (irreducible water), and equivalent resistance of inherent conductive network (including clay, layered clay, conductive minerals and other conductive factors). Wherein, the connected porosity per unit volume, namely the effective porosity of sandstone, is equal to the sum of the pores of free water and those of irreducible water, namely:

$$
\Phi_{\mathrm{e}}=\Phi_{\mathrm{f}}+\Phi_{\mathrm{wi}}
$$

Based on the three-water conductivity model [19, 20] and the principle of equivalent resistance, it is assumed that there are only three curved conductive capillars in sandstone per unit volume, respectively representing free water, irreducible water and inherent conductive network, which belong to non-uniform capillary parallel conduction, while non-connected pore water is considered not to participate in the conduction process. Thus, a rock unit volume conductivity model can be established, as shown in Figure 1.
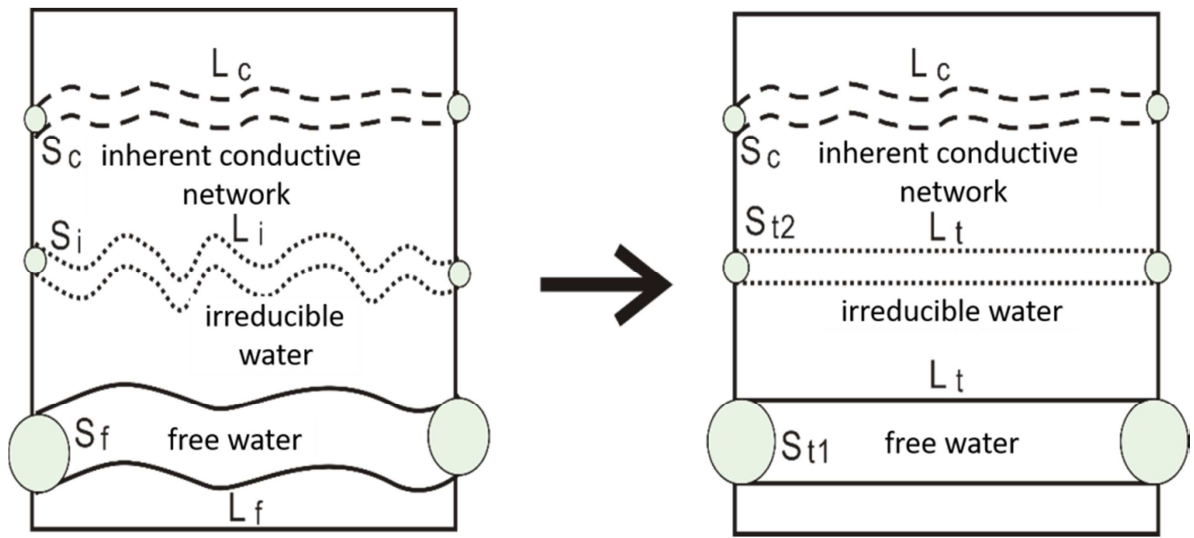

Figure 1. Schematic diagram of nonuniform capillary parallel conduction model per unit volume of rock and its equivalent resistance.

\subsection{Formula Derivation of Unit Volume Model}

According to the unit volume model of sandstone, it is assumed that the sectional area of free water capillary is $\mathrm{S}_{\mathrm{f}}$, length is $\mathrm{L}_{\mathrm{f}}$, the sectional area of irreducible water capillary is $\mathrm{S}_{\mathrm{i}}$ and length is $\mathrm{L}_{\mathrm{i}}$, and the sectional area of inherent conductive network capillary is $S_{c}$ and length is $L_{c}$. See Figure 1 , then the corresponding resistors $R_{f}, R_{i}$ and $R_{c}$ are:

$$
\begin{aligned}
R_{f} & =r_{w} \frac{L_{f}}{S_{f}} \\
R_{i} & =r_{w \mathrm{i}} \frac{L_{\mathrm{i}}}{S_{i}} \\
R_{c} & =r_{\mathrm{c}} \frac{L_{c}}{S_{c}}
\end{aligned}
$$

Then, the resistance per unit volume of rock can be expressed as:

$$
\frac{1}{r}=\frac{1}{\mathrm{R}_{f}}+\frac{1}{R_{i}}+\frac{1}{R_{C}}
$$

It can be seen that micro-pore water (irreducible water) and free water should have the same formation water resistivity, namely $\mathrm{R}_{\mathrm{W}}$, due to the deposition soaking in the same water, so it is not necessary to set up a separate irreducible water resistivity $\mathrm{R}_{\mathrm{WI}}$. Secondly, the curved capillary is not conducive to the derivation of the formula. Instead, equivalent capillary tubes with the same length and different cross-sectional areas can be used, while the total porosity remains unchanged, namely:

$$
\Phi_{\mathrm{e}}=\mathrm{L}_{\mathrm{f}} * \mathrm{~S}_{\mathrm{f}}+\mathrm{L}_{\mathrm{i}} * \mathrm{~S}_{\mathrm{i}}=\mathrm{L}_{\mathrm{t}} * \mathrm{~S}_{\mathrm{t} 1}+\mathrm{L}_{\mathrm{t}} * \mathrm{~S}_{\mathrm{t} 2}
$$

It is assumed that the equivalent capillary length of different cross-sectional areas of the same length is $\mathrm{Lt}$, the free water cross-sectional area and the irreducible water cross-sectional area are St1 and St2 respectively, as shown in Figure 1. In this way, the capillary resistance of free water and irreducible water per unit volume of the rock can be expressed as:

$$
R_{f}=r_{w} \frac{L_{f}}{S_{f}}=\mathrm{r}_{w} \frac{L_{t}}{S_{t 1}}
$$




$$
R_{i}=r_{w} \frac{L_{i}}{S_{i}}=\mathrm{r}_{w} \frac{L_{t}}{S_{t 2}}
$$

According to the principle of parallel conduction, the resistance of sandstone containing water per unit volume can be expressed as:

$$
\frac{1}{\mathrm{R}_{o}}=\frac{1}{r_{w} \frac{L_{t}}{S_{t 1}}}+\frac{1}{r_{w} \frac{L_{t}}{S_{t 2}}}+\frac{1}{R_{c}}
$$

When the reservoir contains oil, the conductive capillary tube length remains unchanged, but the water-bearing sectional area becomes smaller. Because most of the pore volume is oil and gas, assuming that the water-bearing sectional area is St4, the resistance of sandstone per unit volume can be expressed as:

$$
\frac{1}{\mathrm{R}_{\mathrm{t}}}=\frac{1}{r_{w} \frac{L_{t}}{S_{t 4}}}+\frac{1}{r_{w} \frac{L_{t}}{S_{t 2}}}+\frac{1}{R_{c}}
$$

According to formula 11, then:

$$
\frac{1}{\mathrm{R}_{\mathrm{t}}}=\frac{S_{\mathrm{t} 4}+S_{t 2}}{r_{w} L_{t}}+\frac{1}{R_{c}}=\frac{\left(S_{\mathrm{t} 4}+S_{t 2}\right) * L_{t}}{r_{w} L_{t} * L_{t}}+\frac{1}{R_{c}}=\frac{\Phi_{w}}{r_{w} L_{t} * L_{t}}+\frac{1}{R_{c}}
$$

$\Phi_{\mathrm{w}}$ for the remaining water pore volume in the connected pore (remaining free water + micro pore water), besides $\Phi_{\mathrm{w}}$ as a variable, the rest are constant, so formula 12 can be represented as:

$$
R_{t}=\frac{c}{\Phi_{w}}+d
$$

Where $R_{t}$ is resistivity of rock unit volume, units, $\Omega, \mathrm{m}$; $\Phi$ ${ }_{\mathrm{w}}$, the remaining pore water volume in the connected pore, unit, the decimal; $\mathrm{C}$ and $\mathrm{D}$ are coefficients and dimensionless.

Formula 13 is the formula form of the resistivity of sandstone unit volume, which shows that the resistivity of sandstone and the water-bearing pore volume in an ideal state is a simple power function.

It can be seen that the rock resistance is only a function of the remaining water-bearing pores (including micro-pore water) in the effective pores, When the reservoir contains different degrees of oil and gas, the contribution of clay adsorbed water and other inherent conductive factors to the reservoir conductivity does not change, and the fundamental cause of reservoir resistivity change is the change of pore volume of free water. Therefore, the resistivity of oil-bearing sandstone is the change of resistivity based on clay adsorption water and other inherent conductive factors. That is, for the same reservoir, the difference in resistivity between oil and water is based on a resistivity base, which is the contribution of clay adsorption water and other inherent conductive factors to the resistivity. Therefore, the influence of clay adsorption water and other inherent conductive factors on the resistivity change is fixed and can be treated as a constant influence. the oil-gas resistivity response equation can be viewed as a function of the resistivity and the water-bearing pore volume in the connected pores of the reservoir, namely:

$$
\mathrm{R}=\mathrm{F}\left(\Phi_{\mathrm{w}}\right)
$$

How to determine the resistivity formula of sandstone in practice? Due to the non-meanness of real sandstone, the resistance per unit volume in different areas of a sandstone may be different, so the Rt per unit volume in different areas may be different, but the resistance of sandstone is still composed of these resistors per unit volume in series and parallel. Formula 14 can be expressed as:

$$
\frac{1}{R}=\sum_{i=1}^{i=n} \frac{1}{\sum_{k=1}^{k=n}\left(\frac{c_{i k}}{\Phi_{w i k}}+d_{i k}\right)}
$$

Since $\mathrm{c}_{\mathrm{iK}}$ and $\mathrm{d}_{\mathrm{ik}}$ are both constant, it indicates that the rock resistance is still a function of the remaining water-bearing pores (including micro-pore water) in the effective pores, and this function is a composite power function.

According to the above analysis, there are two new ideas: (1) The resistivity of sandstone can be expressed as a function of the connected water-bearing pore volume of rock; (2) The formula form of sandstone resistivity can be expressed as power function relation. According to this principle, a large number of rock electrical measurement data of a single rock sample are counted. According to the measurement results of a single rock sample, the measured resistivity and the water-bearing pore volume were taken for regression. The results showed that the sandstone resistivity and the water-bearing pore volume were a power function, and the correlation coefficient was as high as 0.99 . See Figure 2. The form of this relationship is:

$$
R_{t}=\frac{a R_{w}}{\Phi_{w}^{m}}
$$

Where: Rt - sandstone measuring resistivity, $\Omega, \mathrm{m}$; Rw sandstone formation water resistivity, $\Omega, \mathrm{m} ; \Phi_{\mathrm{w}}$ - pore water volume of sandstone in the effective pore, decimal; A, M regression coefficient, dimensionless.

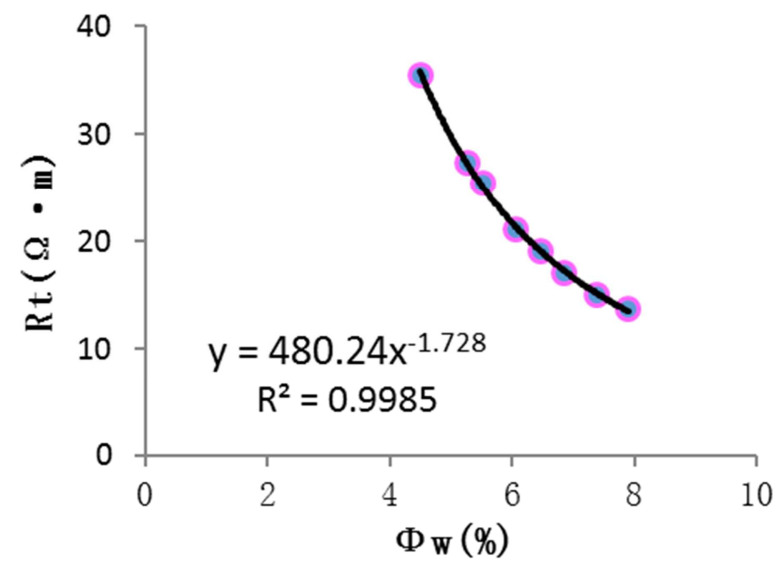

Figure 2. Regression relationship of measurement data of single block rock sample.

Formula 16 is the new resistivity response equation of the oil-bearing sandstone, indicating that the rock resistivity varies with the volume of connected pore water contained in the rock (including micro pores), showing a power function relationship. Therefore, rock resistivity can be expressed as a 
function of the connected pore water resistivity (formation water resistivity) and the pore volume of the connected pore water. The influence of other conductive factors such as clay water and conductive minerals can be reflected in the regression coefficient.

At the same time, the relationship between sandstone resistivity and water saturation is also a power function, which can be derived from the above formula 16. According to the sandstone volume model, the water saturation $S_{w}$ can be expressed as:

$$
\mathrm{S}_{W}=\frac{\Phi_{w}}{\Phi}
$$

According to the formula of 16 , both sides divided by $\Phi^{\mathrm{m}}$, are:

$$
R_{t}=\frac{a R_{w}}{\Phi_{w}^{m}}=\frac{\frac{a R_{w}}{\Phi^{m}}}{\frac{\Phi_{w}^{m}}{\Phi^{m}}}=\frac{\frac{a}{\Phi^{m}} R_{w}}{\left(\frac{\Phi_{w}}{\Phi}\right)^{m}}=\frac{b R_{w}}{S_{w}^{m}}
$$

Then relationship between resistivity and water saturation is:

$$
R_{t}=\frac{b R_{w}}{S_{w}^{m}}
$$

Where, $\mathrm{S}_{\mathrm{w}}$ is water saturation, $\mathrm{B}$ is coefficient, others are the same as equation 16. It shows that the relationship between sandstone resistivity and water saturation is also a power function, and its index is still a pore structure index, not another saturation index.

\section{Analysis of the Coincidence with the Experimental Data and Derivation of the Application Formula}

The data of more than 30,000 rock electric experimental data samples from different blocks of several oil fields were regresated by formula 16 , and the correlation coefficient was not less than 0.99, which reflected the perfect fit between the formula and data. It can be seen that there is no inconsistency in formula 16 as long as the experimental data of rock electricity are reliable. The power function relationship between rock resistivity and water saturation can be fully verified by testing the formula with experimental data. Of course, this relationship has existed for a long time, and researchers should have found that this relationship cannot play an effective role in practical applications because reliable parameters $\mathrm{A}$ and $\mathrm{M}$ cannot be obtained in practical applications. Therefore, empirical formulae such as Archie's formula have always been dominant in determining reservoir water saturation, and this relationship has been selectively ignored by researchers. However, it is not the same now. According to this new formula, a new formula for calculating the water saturation of a reservoir based on the ratio of radial resistivity can be deduced [22]. At present, the formula has been widely used and achieved good results. It can effectively identify the difficult logging layers such as low resistivity oil layer and high resistivity water layer. It shows that it is time to have a deep understanding of the nature of the rock-electric relation. Only by expressing the true relation can we contribute to the further development in the future.

The derivation process of calculating reservoir water saturation by using radial resistivity ratio is as follows, According to Formula 16, the deep resistivity of the reservoir and the flushing zone resistivity can be expressed as:

$$
R_{\mathrm{t}}=\frac{a R_{w}}{\Phi_{w}^{m}} R_{\mathrm{x} o}=\frac{a R_{m f}}{\Phi^{\mathrm{m}}}
$$

Then radial resistivity ratio is:

$$
\frac{\mathrm{R}_{t}}{R_{x o}}=\frac{R_{w}}{R_{m f}} * \frac{\Phi^{m}}{\Phi_{w}^{m}}=\frac{R_{w}}{R_{m f}} * \frac{1}{S_{w}^{m}}
$$

Then water saturation is:

$$
\mathrm{Sw}=\sqrt[m]{\frac{R w / R m f}{R t / R x o}}
$$

Therefore, the radial resistivity ratio can be used to calculate the water saturation of a reservoir. Its advantages and applications have been described in the relevant articles, and will not be repeated here. It should be emphasized that the power function equation of connected pore water volume is used to express the rock resistivity response equation, which can grasp the resistivity variation law of oil-bearing sandstone and reflect the essence of rock conductivity.

\section{The Physical Meaning of Parameter and Its Innovation}

\subsection{Parametric Physical Meaning}

According to the new resistivity response equation formula 16 , the $R_{t}$ is the resistivity of formation rock, and is the resistivity measured by instrument. Rw is the formation water resistivity, which can be reversely calculated from formation water analysis data or adjacent water layer data. $\Phi_{\mathrm{w}}$ is sandstone connected pore water volume, it is need to compute the result. Therefore, there are only two parameters to be determined in practice, namely, regression coefficients A and $\mathrm{M}$ of the data measured by a single block of rock electricity, and the physical meaning of the parameters refers to the physical meaning of the values of A and M. So let's look at M, and by the formula, $\mathrm{M}$ is an exponent of a power function. In view of this formula, $M$ value reflects the degree of influence of the change of connected pore water on the change of rock resistivity. It shows that, with the big $M$ value, the greater the $R_{t}$ value changing with value is larger. The greater the change of resistivity with $c \Phi_{\mathrm{w}}$ onnected pore water, the more heterogeneous the rock is. Therefore, the size of $M$ value can reflect the degree of reservoir heterogeneity. The smaller the $M$ value is, the weaker the reservoir heterogeneity is, and the larger the $\mathrm{M}$ value is, the stronger the reservoir heterogeneity is.

And the value of A, of course, is a direct proportionality 
coefficient, The greater the value of $\mathrm{A}$, the greater the resistivity, and the smaller the value of $\mathrm{A}$, the lower the resistivity. It can be called the increase rate of reservoir resistivity with the change of connected pore water. It shows that for different pore structure lithology, the increasing multiples of resistivity with the change of water-bearing pore volume are different.

To sum up, the $A$ value and $M$ value of the formula are intrinsic property parameters of the rock itself. For the same rock sample, the regressive parameters are the same after immersed rock by using different salinity formation water. It shows that the set of parameters is unique and stable, and can describe the conductivity of rocks.

\subsection{Innovation Ideas}

Compared with previous understandings, this study has five innovations, specifically as follows:

(1) The equivalent resistance is used to simplify the derivation of rock resistivity formula, which is manifested in two aspects: First, equivalent resistance is used to replace the inherent conductive network of clay and conductive minerals to avoid the influence of its complexity on formula derivation. First, equivalent resistance is used to replace the inherent conductive network of clay and conductive minerals to avoid the influence of its complexity on formula derivation. Second, the equivalent capillary tubes with different cross-sectional areas and the same length are used to replace the capillary tubes with different cross-sectional areas and different lengths, which simplifies the derivation of the formula. Therefore, the volume of connected pore water can appear in the expression of unit volume resistance, which provides a basis for further understanding of the influence of connected pore water on conductivity.

(2) The macropore connected water and micropore connected water (irreducible water) are considered to have the same formation water resistivity. However, previously, it was believed that the resistivity of irreducible water was different from that of formation water [23], with special expressions of $\mathrm{R}_{\mathrm{wi}}$. The new understanding is based on the principle of rock deposition and has certain theoretical basis, which reduces the difficulty of formula derivation.

(3) A new conductive theory of connected pore water is proposed, which combines the advantages of parallel conductive between formation water and bound water.

The new formulation avoids the unclear concepts of porosity and water saturation of Archie's formula [24, 25]. Whether the porosity of Archie's formula is total porosity or effective porosity has not been determined, Whether the water saturation is the total water saturation is also controversial, This study addresses that question. The porosity of the new formula is undoubtedly that of all connected pores, including connected micro-pores, which should be the effective porosity of rocks. The corresponding water saturation shall be the total water saturation in the effective pores, that is, including micro-pore water saturation (bound water saturation). In this way, the calculated oil saturation is generally not more than $80 \%$, which provides a basis for the rationality of the test results.

(4) The relationship between rock resistivity and pore volume or water saturation of reservoir connecting water is put forward as a power function, and the relationship between the radial resistivity ratio and water saturation is also a power function, which provides a basis for further research on rock resistivity in the future.

(5) The view that parameter $A$ and parameter $M$ value of the new formula are inherent property parameters of rocks indicates that the lithology of reservoirs can be further divided according to A value and $\mathrm{M}$ value, which opens up a broad application prospect for lithology identification and reservoir evaluation.

\section{Conclusions}

(1) The derivation process of the new rock resistivity response equation is reasonable, which is in good agreement with the experimental data of well logging rock electricity, and the correlation coefficient of the regression formula is high, which avoids the limitation of large relative error between archie formula and the experimental data. It can reflect the nature of rock conductivity, and can be popularized in practice.

(2) According to the new formula, the relationship between rock resistivity and connected pore water volume or water saturation is a power function, and the relationship between rock radial resistivity ratio and water saturation is also a power function. This relationship is more simple and practical, which is a new concept and completely changes the current understanding of logging interpretation engineers on rock resistivity response equation.

(3) The new rock resistivity response equation provides a theoretical basis for further understanding the rock-electric relationship and improving the identification accuracy of well logging oil and water layers, and provides a new means for the evaluation of low resistivity oil layers and other difficult layers.

(4) There is no better method to determine the parameters of the new formula except using the experimental data of rock electricity, which needs to be further studied in the future.

\section{References}

[1] Archie, G. E. The electrical resistivity $\log$ as an aid in determining some reservoir characteristics [J]. Tnas Am Inst MechEng, 1942, 146: 54 61.

[2] Simandoux P. Dielectric measurements of porous media: Application to measurement of water saturations, study of the behavior of argillaceous formations [J]. Revue de L, Institut Franais du Petrole, 1963, 18 (S1): 193 215.

[3] Mao Zhiqiang, Gong Huhua, Liu Changyu, et al. Experimental study on the gensis of low resistivity pay zone in north region of Tarim basin (I) [J]. Well Logging Technology, 1999, 23 (4): 243-245. 
[4] Zhao Yanchao, Zhou Boran. Study on three kinds of conductivity models for shaly sand formations [J]. Well Logging Technology, 1998, 22 (2): 75-78.

[5] Sun Jianmeg, Wang Kewen, Li Wei. Development and analysis of logging saturation interpretation models [J]. Petroleum Exploration and Development, 2008, 35 (1): 101-107.

[6] Zhang Lihua, Pan Baozhi, Li Zhoubo, et al. The three-Water conductive model and its application in the evaluation of low-porosity and low-permeability reservoirs [J]. Oil Geophysical Prospecting, 2010, 45 (3): 431-435.

[7] Song Yanjie, Chen Jiqiang, Tang Xiaoming, et al. Three-water pore combination conductive model for low porosity and permeability shaly sand and its application to offshore oilfields [J]. Journal of Daqing Petroleum Institute, 2010, 34 (6): 106-110.

[8] Mao Zhiqiang, Zhi Weihong, Wang Rujun. Experimental study on the gensis of low resistivity pay zone in north region of Tarim basin (II) [J]. Well Logging Technology, 1999, 23 (6): 404-410.

[9] Zhao Yanchao, Wu Chunping, Wu Dongping. Logging evaluation to tight gas sandstone: a case study from the firstmember of shanxi formation in daniudi gas pool, ordos basin, china $[\mathrm{J}]$. Geological Science and Technology Information, 2003, 22 (4): 65-70.

[10] Sheng Aixin, Wang Li, Chen Shoujun. Low reservoir resistivity and litho-electric experiment of each parameter in Archie equation [J]. Journal of Jianghan Petroleum Institute, 2003, 25 (suppl.): 24-25.

[11] Zhang Minlu, Shi Yujiang. On Archie's electrical parameters of sandstone reservoir with complicated pore structures [J]. Well Logging Technology, 2005, 29 (5): 446-448.

[12] Sun Jianguo. Archie's formula: historical background and earlier debates [J]. Progress in Geophysics, 2007, 22 (2): $472-486$.

[13] Yang Kebing, Wang Jinfei, Ma Fengqin, et al. Analysis and Countermeasures about applicable conditions of Archie's Formula [J]. Natural Gas and Oil, 2018, 36 (2): 58-63.

[14] Zhang Jie, Luo Jian, Xia Yu. Limitation analysis and modification of the Achie equation [J]. Chinese Journal of Geophysics, 2018, 61 (1): 311-322.
[15] An analysics of the factors to influence electrical properties of rocks based on a digital petrophysical experiment [J]. China offshore oil and gas, 2012, 24 (6): 12-16.

[16] Zhang Zhaohui, Gao Chuqiao, Gao Yongde. Theoretical simulation and analysis factors of resistivity in vuggy reservoir $[J]$. Journal of Southwest Petroleum University (Science \& Technology Edition), 2014, 36 (2): 79-83.

[17] Zhang Jianhua, Liu Zhenhua, Wu Jie. Principle and application of electrical logging [M]. XiAn, Northwestern University Press, 2002.

[18] Yong Shihe, Zhang Chaomo. Log data processing and comprehensive interpretation [M]. DongYing, China University of Petroleum Press, 2007.

[19] Mo Xiuwen, He Duohua, Li Zhoubo, et al. The application of three -water conduction model in the interpretation of low-resistivity reservoir [J]. Journal of Changchun University of Science and Technology, 2001, 31 (1): 92-92.

[20] Ding Haiming, Wu Xiaoli. Research on logging interpretation methods for 3 Porosities in muddy sandstone [J]. Petroleum Geology \& Oilfield Development in Daqing, 2005, 24 (4): 101-102.

[21] Song Yanjie, Zhang Yanan, Tang Xiaomin, et al. A conductivity model for dolomite reservoirs based on connectivity theory [J]. Journal of Harbin University of Commerce (Natural Sciences Edition), 2015, 31 (2): 247-252.

[22] Yang Kebing, Xi Li, Zhang Lei, et al. Study on a New Method for Calculating Water Saturation [J]. International Journal of Oil, Gas and Coal Engineering, 2019, 7 (6): 125-128.

[23] He Ling, Ding Yujiao, Chi Xiurong, et al. On quantitative qaturation evaluation method for internal cause low resistivity reservoirs in Qikou Sag [J]. Well Logging Technology, 2014, 38 (1): $51-58$

[24] Jing Wanxue, Chen Yongji. On the Original Physical Model of Archie Formula [J]. Well Logging Technology, 1997, 21 (4): 289-291.

[25] Yang Kebing, Yuan Xiaohong, Liu Mingbo, et al. Resistivity ratio method to evaluate oil-water layer [J]. Natural Gas Exploration \& Development, 2015, 38 (1): 37-39. 